\title{
Pornographic content classification using deep-learning
}

\author{
André Tabone \\ Systems \& Control Engineering \\ University of Malta, Malta \\ andre.tabone@um.edu.mt \\ Stefania Cristina \\ Systems \& Control Engineering \\ University of Malta, Malta \\ stefania.cristina@um.edu.mt
}

\author{
Kenneth Camilleri \\ Systems \& Control Engineering \\ University of Malta, Malta \\ kenneth.camilleri@um.edu.mt \\ Reuben Farrugia \\ Computer Engineering \\ University of Malta, Malta \\ reuben.farrugia@um.edu.mt
}

\author{
Alexandra Bonnici \\ Systems \& Control Engineering \\ University of Malta, Malta \\ alexandra.bonnici@um.edu.mt
Mark Borg
Systems \& Control Engineering
University of Malta, Malta
mborg2005@gmail.com

\begin{abstract}
Controlling the distribution of sensitive content such as pornography has become paramount with the ever-growing accessibility to the internet. Manual filtering of such large volumes of data is practically impossible, thus, the automatic detection of said material is sought after by Law Enforcement Agencies (LEAs) and has been tackled in various manners. However, the sorting of flagged pornographic documents is still done manually using scales that describe hierarchical degrees of content severity. In this paper, we address pornography detection by creating a model capable of locating and labelling sexual organs in images and extend this model to perform image classification to provide the user with one of 19 semantically meaningful descriptors of the content. Generating these descriptors serves as a proof of concept before approaching LEAs to work with illegal CSA material and scales such as COPINE. After creating our own custom sexual organ object detection dataset for the task at hand, we achieved an object detection mean average precision score of $63.63 \%$ and a top-3 classification accuracy of $87.78 \%$.
\end{abstract}

\section{CCS CONCEPTS}

- Computing methodologies $\rightarrow$ Object detection.

\section{KEYWORDS}

Deep learning, Pornography classification, object detection

\section{ACM Reference Format:}

André Tabone, Kenneth Camilleri, Alexandra Bonnici, Stefania Cristina, Reuben Farrugia, and Mark Borg. 2021. Pornographic content classification using deep-learning. In ACM Symposium on Document Engineering 2021 (DocEng '21), August 24-27, 2021, Limerick, Ireland. ACM, New York, NY, USA, 10 pages. https://doi.org/10.1145/3469096.3469867

Permission to make digital or hard copies of all or part of this work for personal or classroom use is granted without fee provided that copies are not made or distributed for profit or commercial advantage and that copies bear this notice and the full citation on the first page. Copyrights for components of this work owned by others than ACM must be honored. Abstracting with credit is permitted. To copy otherwise, or republish, to post on servers or to redistribute to lists, requires prior specific permission and/or a fee. Request permissions from permissions@acm.org.

DocEng '21, August 24-27, 2021, Limerick, Ireland

(C) 2021 Association for Computing Machinery.

ACM ISBN 978-1-4503-8596-1/21/08 . \$ \$15.00

https://doi.org/10.1145/3469096.3469867

\section{INTRODUCTION}

Whilst pornographic material obtained with consent of involved parties is legal, the distribution of unsanctioned content, particularly if children are involved is illegal. When there is any suspicion of creation or distribution of illicit content, Law Enforcement Agencies (LEAs) need to go through large volumes of documents belonging to the suspected perpetrator in order to gather tangible evidence before making a criminal case. In the case of Child Sexual Abuse (CSA), LEAs maintain an international database of image content to help with the tracking of such material. Given such a database, tools in use by LEAs that 'detect' illicit content among a perpetrators documents revolve around hashing algorithms. Whilst being very computationally efficient and robust against generating false positive alarms, hashing algorithms are limited to detecting content identical to that within the LEA's database. Recently, multiple deep-learning frameworks are being developed in an attempt to address this limitation [2, 10, 22]. However, such data driven solutions tend to suffer from high false positive rates or high negative rates when performing CSA detection since the sensitive nature of CSA material makes it difficult to collect large datasets and would normally be carried out in close cooperation with LEAs's. Consequently, the detection of adult pornography is more commonly attempted.

Once seized, the description and classification of the nature of the material is important to extract the full forensic value and appropriately store it into national and international databases [5]. The Combating Paedophile Information Networks in Europe (COPINE) scale [20] is currently the prevalent ranking system used to categorise child abuse images in both legal and research settings [5]. The ten degrees adopted by this scale range from indicative content as the least severe, to sadistic/bestiality as the most extreme case. The Sentencing Advisory Panel (SAP) later reduced these ten levels to five categories referred to either as the SAP scale or the Oliver scale [5]. To help assist courts in weighing the perpetrator's sexual interest in children, the Australian National Victim Image Library (ANVIL) scale includes three additional categories to the SAP scale which are not illegal themselves but are directly connected to the sexual exploitation of children. The three additional categories include cartoons and drawings depicting children engaged in sexual activity, benign exploitation of children, and adult pornography. The current array of deep learning tools aimed at the detection of pornographic content $[11,19,29,31]$ are limited to flagging sensitive content and do not distinguish between different degrees 
of pornographic material. Thus, LEAs do not have the means to automatically categorise and organise the sensitive content seized from the perpetrators.

In this paper, we aim to develop a framework capable of detecting pornographic images and provide a descriptor that enables the users to categorise documents according to the pornographic nature of the content. We adopt Wehrmann et al.'s definition of pornography where the presence of sexual body parts is both textually and visually implied when describing pornographic content [34] Thus, developing algorithms that detect sexual body parts provide LEAs with tools which not only detect pornographic content but also provide descriptors justifying the decision. We improve on Mallmann's private part detector [19] by presenting a solution that detects twice as many object classes with a $270 \%$ increase in image throughput. In addition, we extend this model to also provide a description to image documents that would help in ranking the degree of pornographic explicitness of a file. Due to the sensitive nature of CSA material, we demonstrate a concept framework that labels an image with one of 19 possible class names based on a publicly available adult pornography dataset. The general pornographic descriptions provided by this work are a step towards approaching the automatic classification of images according to scales such as the COPINE scale, since it is necessary to have a working prototype in place such that LEAs may then fine tune on their illicit data.

\section{LITERATURE REVIEW}

PhotoDNA ${ }^{1}$ is considered as the current state of the art CSA detection tool. It is deployed on most of the largest online platforms such as Reddit, Facebook and Twitter. Microsoft started developing this tool in 2009 and this perceptual fuzzy hash technology was deployed on their Azure cloud service in 2015, making it easier and less costly for smaller companies to use. This tool creates a unique digital signature based on the image content called a hash. This hash is compared to a database of hashes obtained from previously identified illegal images. The Internet Watch Foundation (IWF) is responsible for developing the largest hash database ${ }^{2}$ with more than 200,000 hashes having been generated using the PhotoDNA tool or the standard cryptographical Message-digest (MD5) algorithm [35].

Whilst such hashing methods have proven to be reliable in preventing false detections, their main limitation still stands out, namely that these algorithms are unable to respond to new content. In order to mitigate this, automated image understanding is required [15]. In machine learning, feature extraction methods are used to obtain a set of visual properties on which conclusions about the image content can be made. Originally, such feature extraction frameworks were manually engineered as shown by Kejun et al. [11]. In their work they detect female breasts by using nipple shapes and colours as proposed by Wang et al. [33] along with additional Haar-like features as done by Lienhart et al. [16]. Kejun et al. [11] then use these extracted features to train an AdaBoost Classifier as a female breast detector. Unfortunately, such algorithms tend to suffer from false positives where body regions such as the belly-button and eyes generate features that are sufficient similar to those of the nipple. Kejun et al. [11] show that results improve when

\footnotetext{
${ }^{1}$ https://www.microsoft.com/en-us/photodna

${ }^{2}$ https://www.iwf.org.uk/our-services/hash-list
}

including additional Hue, Saturation and Value (HSV) based colour features, namely mean, variance and skewness. For complex tasks, the stacking of multiple feature extraction methods can quickly become taxing and computationally complex.

In recent years, deep-learning algorithms have outperformed most classical feature algorithms that preceded them [7, 17, 24]. Deep learning algorithms require large sets of examples and ground truth outputs from which they automatically learn to extract a larger spectrum of complex and abstract features [2]. Due to the large number of publicly available examples, Convolutional Neural Network $(\mathrm{CNN})$ have become the state of the art algorithms when dealing with adult pornography detection. However, due to the sensitivity and safety restrictions required around CSA material, deep-learning approaches usually do not find enough training data to outperform and justify replacing hashing algorithms. In fact, Vitorino et al. [31] propose a two step transfer learning methodology where they reduce the number of required sexually exploitative imagery of children (SEIC) training data by using an intermediary fine tuning step. They start from a pre-trained model, which they fine tune to perform adult content detection before using a second fine tuning stage to convert the model to a SEIC detector. Using this method they manage to pinpoint images depicting assault, gross assault and sadistic/bestiality involving children, which are considered to be of high importance on the COPINE scale [20]. Moustafa et al. [22] fine tune pre-trained AlexNet and GoogLeNet architectures to detect adult pornography. The Softmax outputs of these two models were assumed to be complementary and combined to improve the accuracy scores of each independent network. However, using multiple models as weak classifiers contributing to an overall strong classification greatly increases the number of computations required, decreasing the throughput of the framework. Instead of using multiple models, Huang et al. [10] introduce a colour feature histogram as an additional input to the CNN. They also integrate bagging into the $\mathrm{CNN}$ to increase the generalisation of the model by reducing over-fitting.

Wehrmann et al. [34] later note that many of the false-positives in deep-learning pornography classification models can be avoided by making sure that exposed sexual organs are actually present in the images. Furthermore, Tran et al. [30] remark that the definition of pornographic content implies that the said images must contain sensitive objects usually male or female genitals, breast and anus. Tran et al. [30] make use of the Mask R-CNN [1] architecture to detect and segment these sensitive objects. A Support Vector Machine (SVM) is used to perform the final binary classification using the number of objects detected per class and their highest confidence. Shen et al. [28] propose an Ensemble Framework using Uncertain Inference (EFUI), which uses a Single Shot MultiBox Detector (SSD) model to identify sensitive semantic components including genital, breast, buttocks, nude body and sexual action. Similarly, Wang et al. [32] bag local image patches containing female breasts and sex organs before using Multiple Instance Learning (MIL) to recognise images as pornographic if they contain at least one sexual organ. Their MIL approach achieved a better accuracy when compared with traditional pornographic classification methods such as image retrieval or bag-of-features. 
Table 1: Method and performance comparison of algorithms in previous literature

\begin{tabular}{|c|c|c|c|}
\hline Paper & Aim & Approach & Performance \\
\hline \multirow[t]{3}{*}{ Kejun et al. [11] } & \multirow[t]{3}{*}{ Female breast detection } & \multirow[t]{3}{*}{ Classical hand-crafted features } & True positive rate: $90.37 \%$ \\
\hline & & & False positive rate (FPR): $7.46 \%$ \\
\hline & & & False negative rate (FNR): $4.86 \%$ \\
\hline Moustafa et al. [22] & Adult Video Pornography detection & CNN models AlexNet \& GoogLeNet & $94.2 \pm 2 \%$ on NPDI- 800 dataset \\
\hline Vitorino et al. [31] & $\begin{array}{l}\text { Detection of sexually exploitative } \\
\text { imagery of children \& adults }\end{array}$ & Colour feature histogram \& GoogLeNet CNN & $\begin{array}{l}91.5 \% \text { accuracy on Adult Ponrnography- } 2 \mathrm{~K} \text { dataset } \\
86.5 \% \text { on a SEIC dataset }\end{array}$ \\
\hline Shen et al. [28] & Adult Pornography detection & SSD extracted features & $94.7 \%$ accuracy on NPDI dataset \\
\hline Tabone et al. [29] & Sexual organ detection & Multi-Class Classifiers and Windowing & $62 \%$ window classification accuracy \\
\hline Tran et al., $2020[30]$ & Adult Pornography detection & $\begin{array}{l}\text { Sexual object detection using Mask-RCNN } \\
+ \text { binary video classification through a SVM }\end{array}$ & $\begin{array}{l}90.125 \% \text { video level accuracy on NPDI- } 800 \\
90.43 \% \text { accuracy on } 40 \mathrm{k} \text { custom test images }\end{array}$ \\
\hline \multirow[t]{3}{*}{ Mallmann et al., 2020 [19] } & Sexual organ detection & Faster R-CNN Inception v2 & $63.5 \mathrm{mAP}-50$ on Adult PPO dataset \\
\hline & Adult Video Pornography detection & Faster R-CNN Inception v2 as a binary classifier & FPR: $1.66 \%$ \\
\hline & & & FNR: $2.34 \%$ on Adult Pornography-2k test set \\
\hline \multirow[t]{2}{*}{ Mahadeokar et al., 2016 [18] } & \multirow[t]{2}{*}{ Adult pornography detection } & \multirow[t]{2}{*}{ Thin ResNet-50 } & FPR: $12.8 \%$ \\
\hline & & & FNR: $5.53 \%$ on Adult Pornography-2k test set \\
\hline
\end{tabular}

AUC(\%): Area Under Curve (AUC) of the Receiver Operating Characteristic (ROC) curve

SEIC: Sexually explitative images of children

PPO: Private Parts Object Dataset

mAP-50: mean average precision metric with an intersection over union threshold of 0.5

Tabone et al 2020 [29] propose a two step ConvNet system that uses a binary MobileNet classifier as an initial pornography detector. A windowing approach combined with a multi-class MobileNet classifier then locates the five main sexual body parts: male genitalia, female genitalia, buttocks and sex toys. They note that the female genitalia displayed within obscene posing images tend to differ substantially from those within sexual activities, thus splitting this class into female genitalia posing and female genitalia active. An additional benign class was used in the multi-class MobileNet to represent the possible background. Mallmann et al. [19] propose an architecture that addresses pornography content detection entirely through object detection. They manually label 52,215 object instances to create their Private Parts Object (PPO) dataset which is used to fine tune a faster R-CNN Inception architecture for real-time object detection. Their final model is capable of detecting male and female sexual organs, female breasts, and buttocks, outperforming the state of the art Yahoo Detector [18].

The automatic description generation for images is a task that has been received by both the Natural Language Processing (NLP) and Computer Vision (CV) communities. Bernardi et al. [4] split the automatic image description models into three categories. The first approach utilises computer vision techniques to identify objects, their attributes and the relationships they hold with the surrounding environment. The extracted information is then used to generate phrases which can then be further refined through natural language generation. When feature extraction is tailored for a specific application as seen in Kulkarni et al. [13], the generated descriptions are constrained to exist within a predefined set of semantic classes. Description generation has also been approached as a retrieval task, where frameworks use the visual space to find similar images and transfer descriptors to the queried image. Such systems require a large amount of training data [4]. Delvin et al. [6] use a $\mathrm{CNN}$ to extract image features and perform k-nearest neighbour retrieval form their training set to find candidate descriptions. The last approach reviewed tackles the retrieval problem in the Multimodal space, where both an image and sentence features are extracted from the queried image before performing the retrieval task. The advantage here is that bi-directional models can be used to for the other direction, thus retrieving an image for a queried sentence $[8,12,14]$

Table 1 outlines the aims and results achieved by the reviewed methods from which we note that there has not yet been established a standard set of explicit object classes and a corresponding benchmark dataset. Furthermore, listing the sexual organs found in images is not enough to describe the severity of pornographic content in an image as a whole. To our knowledge there is no framework that tackles this problem directly.

\section{METHODOLOGY}

To aid law enforcement agencies automate their pornography detection and filtering processes, we aim to develop a sexual organ detection system that is both fast and accurate. Our proposed system is also intended to provide a class label which describes the degree of explicitness of the image content. In this section we will address the limitation of freely available pornographic object detection data by creating our own sexual organ detection dataset. We follow this by describing the training approach taken to obtain our own sexual organ detector. In Section 3.4, we propose a method which targets the object class imbalance within our dataset, by applying a custom confidence threshold per class during inference. This improves the performance of the model and provides the user with a parameter that can vary the precision and recall of the model. Finally, we demonstrate how the features learnt by this model can be used to efficiently perform an additional task, namely image classification.

\subsection{Sexual Object Detection Dataset}

When detecting object with irregular shapes, systems used in autonomous driving implement semantic segmentation rather than normal box detection [9]. However, image segmentation takes significantly more work to label and since we do not prioritise the precise localisation of objects, we stick to normal box annotations. 


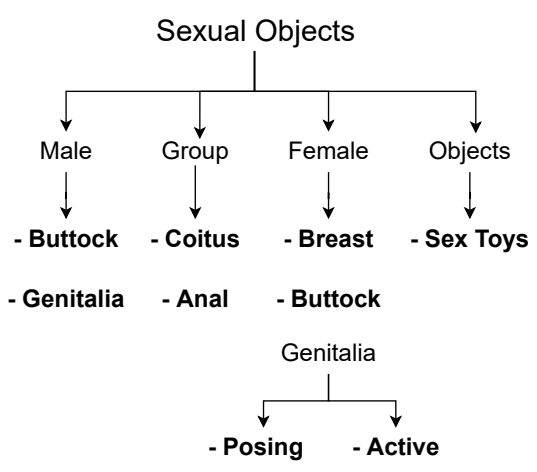

Figure 1: Objects that make an image pornographic

Table 2: Dataset generated

\begin{tabular}{l|r|r|r|r}
\hline \multirow{2}{*}{ Class Name } & \multicolumn{2}{|c|}{ Training } & \multirow{2}{*}{ Testing } & \multirow{2}{*}{ Hold Out } \\
\cline { 2 - 3 } & Pre Aug & Post Aug & & 224 \\
\hline \hline Female Breast (FB) & 13025 & 24668 & 1301 & 98 \\
Male Genitalia (MG) & 3937 & 13880 & 359 & 58 \\
Female Buttock (FBT) & 2841 & 7058 & 284 & 40 \\
Coitus (C) & 1480 & 10247 & 107 & 11 \\
Anal (A) & 235 & 1616 & 24 & 15 \\
Male Buttock (MBT) & 509 & 2426 & 32 & 69 \\
Female Genitalia Active (FGA) & 3377 & 8108 & 307 & 20 \\
Female Genitalia Posing (FGP) & 1500 & 1564 & 170 & 10 \\
Sex Toys (ST) & 717 & 789 & 78 & 545 \\
Total & 27621 & 70356 & 2662 & \\
\hline
\end{tabular}

Aug: Augmentation

Consistency in an object detection dataset is fundamental for any model being trained to correctly learn how to discriminate between foreground/background and object classes. We note, however, that in pornographic images, there are multiple instances where, due to the nature of pornographic images, sexual organs share visual traits with other body parts and even overlap with other sexual organs. This introduces an element of subjectivity in the groundtruth labelling, where it is at times difficult to decide whether an object is too occluded to be labelled. In our approach, we avoid overlabelling such instances as they introduce false positives in benign images such as beach photos, water sports, underwear catalogues among others, where large skin exposure is expected.

To create our sexual organ detection dataset we first establish the set of objects that make images sexually explicit. The selected object classes are shown in Figure 1 which depicts how we outline the four sources of pornographic content as Male, Female, Groups, and Objects. The object classes to be detected were based on what explicit content each source generates to obtain a total of eight classes. It was noted that "Female Genitalia" should be split into two subsidiary classes since the appearance differs considerably between instances of posing and instances of sexual activity [29]. Since eight out of our nine classes are essentially body parts, they are expected to share a number of features. This reinforces the importance of annotating boxes in a manner where the minimum possible area is highlighted whilst making sure that the important visual cues such as the nipple for "Female breast" are not covered.

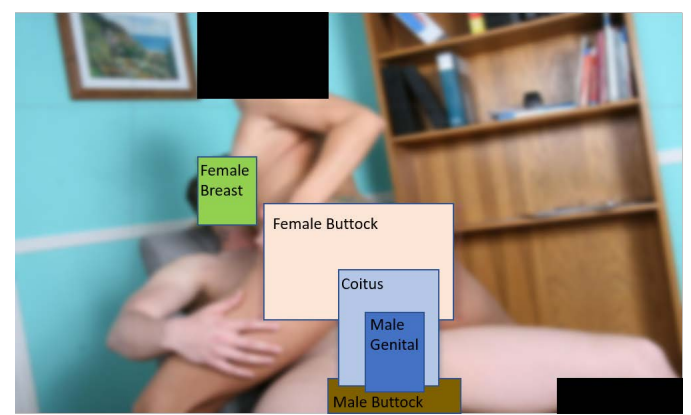

(a)

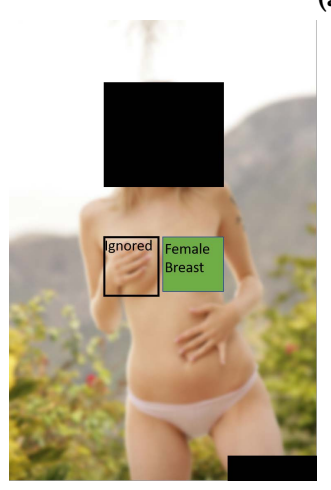

(b)

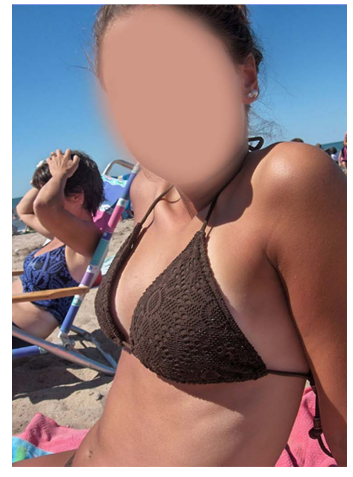

(c)
Figure 2: Labelling criteria showing (a) High overlap between object classes, (b) Ignoring partially occluded instances (c) Benign images with high skin exposure around organ areas that may cause false positives

Figures $2 \mathrm{~b}$ and $2 \mathrm{c}$ illustrate an example pair where a partially covered female breast is not labelled in Figure $2 \mathrm{~b}$ since the nipple region is not visible. Even though the whole image may be considered as explicit, by not labelling the covered breast instance during labelling, we prevent a high incidence of breast detections in benign images with similar skin exposure around the said breast region such as that shown in Figure 2c. Furthermore, classes "Coitus" and "Anal" are used to imply the presence of subsidiary object classes which might be occluded during explicit acts. Figure 2a illustrates how labelling the "Coitus" class relieves the need to label the partially occluded "Female genitalia" class. Thus, rather than cluttering the "Female genitalia" class with weak examples, we instead imply its presence through the more distinguishable "Coitus" class. Meanwhile, if easily distinguishable, subsidiary classes are still labelled, as is the case of "Male Genitalia" in this example. Furthermore, the ninth "Sex Toys" class refers to a wide variety of objects whose appearance is easily correlated to sexual content. However, we do not to label sexualised everyday objects to prevent false positives emerging from identical objects in benign contexts.

Two female and three male annotators whose age ranges from 23 to 53 years, with a median age of 38 years provided annotations for the data set. Four of the five annotators have experience in forensic research, which they used to set annotation ground rules. A subset of the large-scale pornographic image corpus created by the 
Table 3: Dataset used

\begin{tabular}{lrr}
\hline & Number of Images \\
\cline { 2 - 3 } Class Name & Training & Testing \\
\hline Benign Image (BN) & 695 & 77 \\
Dressed Partially (DP) & 799 & 88 \\
Models Posing Alone (MPA) & 1096 & 120 \\
Models Posing Group (MPG) & 94 & 8 \\
Dressed Sexual Interaction (DSI) & 19 & 2 \\
Sexual Posing Alone (SPI) & 3,609 & 401 \\
Sexual Posing Group (SPG) & 733 & 63 \\
Exposed Genitals Posing Alone (EGPA) & 2,353 & 261 \\
Exposed Genitals Posing Group (EGPG) & 915 & 101 \\
Fellatio (F) & 1,693 & 187 \\
Cunnilingus and Anilingus (CA) & 566 & 46 \\
Intercourse Fully Naked Couple (IFNC) & 1,797 & 199 \\
Intercourse Partially Naked Couple (IPNC) & 936 & 104 \\
Intercourse Fully Naked Group (IFNG) & 570 & 39 \\
Intercourse Partially Naked Group (IPNG) & 305 & 29 \\
Sexual Interaction Partially Naked Couple (SIPNC) & 974 & 104 \\
Sexual Interaction Fully Naked Couple (SIFNC) & 515 & 51 \\
Sexual Interaction Partially Naked Group (SIPNG) & 272 & 5 \\
Sexual Interaction Fully Naked Group (SIPNG) & 285 & 13 \\
Total & 18,226 & 1898 \\
\hline & & \\
\hline
\end{tabular}

University of Leon (UL) [23] ${ }^{3}$ was provided to each annotator to be independently labelled using Microsoft's Visual object Tagging Tool (VoTT) [21]. Since some class instances are prone to subjectivity, labelling fatigue may introduce inconsistencies and labelling errors. Hence, the resulting 10, 072 images and 30,828 annotations were verified by one of the annotators to ensure consistency. The data detailed in Table 2 has been made publicly available through Group for Vision and Intelligent Systems (GVIS) ${ }^{4}$.

\subsection{Classification Dataset}

The goal of the work carried out in this section and Section 3.5 is to deliver LEAs with image level descriptions that can be used as a tag to rank, sort and filter large volumes of pornographic documents. The image corpus that was created by the UL [23] also included a subset of images that were manually given a category that distinguishes between different possible pornographic scenarios found in image documents. Each image in this labelled subset contained one of 19 possible categories. These categories were formulated around three attributes namely; the number of people involved, the degree of nudity, and the acts being performed by the people within the content. The 19 scenarios used are outlined in Table 3, where we also show the number of images belonging to each scenario along with how the examples were split for training and testing. By distinguishing between these scenarios, our concept framework would be exhibiting a high enough level of understanding that encourages its applicability to standardised scales such as the COPINE or SAP.

\subsection{Sexual Object Detection Model}

Due to the nature of the content, some classes appear more frequent than others since they are common to a larger spectrum of pornographic scenarios. This is most evident in the over representation

\footnotetext{
${ }^{3} \mathrm{http}: / /$ gvis.unileon.es/dataset/apd-2m/

${ }^{4}$ http://gvis.unileon.es/dataset/sexual-organ-detection-sod/
}

of the "Female Breast" class, where irrespective of the volume of data collected, we expect such imbalances to remain present. To overcome this unbalance, we perform six standard data augmentations on images containing the two most heavily under represented classes, namely "Anal" and "Male Buttock".

Table 2 shows the number of usable class examples after data augmentation. Note that although we apply the data augmentation to a select set of images that contain the under-represented classes, these images may contain instances of other classes, hence the unintentional increase in the number of over-represented classes.

The YOLOv3 real-time object detector [25] was selected as our main architecture since it can be trained quickly using the whole image as additional context for the prediction of boundaries which results in fewer false positives when compared to its closest competitor the faster-RCNN [26]. The image input size of $416 \times 416$ was selected as a compromise between minimising the number of computations per inference and maximising the precision of the model. At this size, Redmon et al. reported that the YOLOv3 obtained a mean Average Precision 50 (mAP-50) score of 55.3 with an inference time of $29 \mathrm{~ms}$, in comparison to the 57.5 obtained by the RetinaNet-101-800 in 198ms [25].

To increase the generalisation of the Sexual object detection (SOD) model and decrease the number of false positives emerging from benign images, we split our training regime into two stages and collect two sets of weights. Our first stage loads a set of pretrained ImageNet weights and freezes the image feature extraction portion of the YOLOv3 model. This results in the object detection portion of the model to be trained on general image features learnt by the DarkNet-53 base model on the ImageNet dataset ${ }^{5}$. The number of parameters to be adjusted is also reduced, speeding up the training process. We use a batch size of 64 , early stopping with a patience of ten, drop learning rate on plateau by a factor of 0.1 with a patience of three, Adam optimiser with an initial learning rate of $10^{-4}$ and a maximum number of 51 epochs. Once the first stage finishes, the best obtained weights are kept according to the validation loss. The second stage unfreezes the current model and continues with end to end training using a batch size of six whilst keeping the other parameters unchanged. Again, the best set of weights are kept according to the validation loss.

This model outputs a large number of candidate boxes per image, which are filtered using a Non-Max Suppression (NMS) algorithm, where boxes with confidence scores lower than a set threshold are removed. The remaining are grouped according to their class. Instances where multiple boxes of the same class overlap are reduced by keeping the candidate box with highest confidence. In our specific application, whilst most classes can exist overlapping each other, in which case overlapping outputs have to be permitted, the "Female Genitalia" class is split into two sub-classes representing two possible appearances, thus, the boxes for these two classes are mutually exclusive. Therefore, the NMS is modified to jointly consider and suppress overlapping instances of this class pair.

\subsection{Multi-Confidence thresholding}

Even though data augmentation was performed, improving the representation of the under-represented classes, the nature of the image

\footnotetext{
${ }^{5}$ http://www.image-net.org/
} 


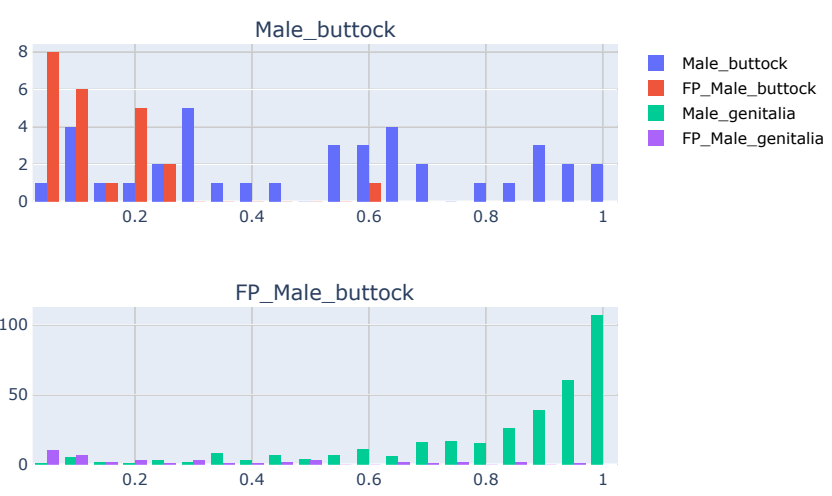

Figure 3: True positive vs False positive example histograms

content still preserved the over representation of some classes. We hypothesise that since our model was exposed to some classes more than others, different classes are expected to have different confidence ranges. Therefore, setting a single confidence threshold for all classes will create a bias against some classes. In this Section we describe a methodology that attempts to extract any class confidence biases exhibited. In doing so, we aim to obtain a vector of optimal class specific confidence thresholds that will replace the single confidence threshold set in the original inference.

A small holdout set of 176 pornographic images was set aside when creating the main dataset. To represent how the model suppresses false positives in non-pornographic images, we add 50 benign images to this hold out set. We proceed to infer the class labels for these 226 images at a very low confidence threshold of 0.05 and collect a set of potential predictions. These potential predictions are compared to their corresponding annotations and marked as either true positives, false positives, false negatives or misclassifications. Figure 3 illustrates the distribution of false positives against true positives for two classes obtained from the test set. Using this illustration it becomes evident that the optimal confidence threshold for each class is different and can also depend on the user's preference towards prioritising either the precision or recall of the model.

In order to determine a suitable confidence threshold for each class, the hold out set is used to generate Receiver Operating Characteristic (ROC) curves for each class, and class confidence thresholds are chosen such as to maximise the weighted F1 score defined in Equation 1. The weighted F1 score uses $\alpha$ as a user-chosen parameter to favour recall over precision as $\alpha$ increases; for an $\alpha=0.5$, the weighted F1 score reduces to the standard F1 score.

$$
F 1_{\text {score }}=2 * \frac{(\alpha * \text { Precision }) *((\alpha-1) * \text { Recall })}{(\alpha * \text { Precision })+((\alpha-1) * \text { Recall })}
$$

\subsection{Sexual Content Classification Model}

A brief overview of the architecture trained in Section 3.3 is shown in Figure 4. The YOLOv3 can be said to contain three main blocks, the first of which is a custom CNN architecture called DarkNet53 '(a)' that extracts image features and acts as the backbone of the model. Three different sizes of feature maps are then used to perform object detection '(b)' through additional CNN layers. The

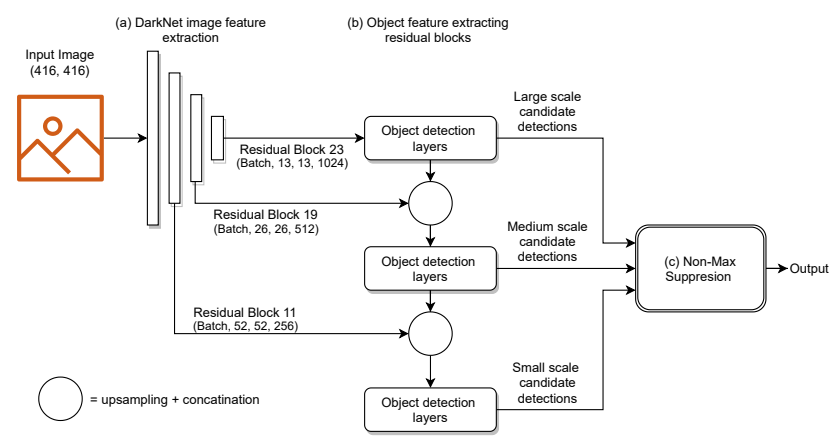

Figure 4: Sexual object detection architecture

candidate boxes proposed by the model are finally passed to a non-max suppression stage '(c)' which generates the final result.

Typically, such a classification task would require the use of a dedicated CNN network. A lightweight architecture like the MobileNetV2 [27] would still require at least an additional two million parameters to extract enough image features to successfully perform the task. However, instead of training a new classifier from scratch, we hypothesise that our SOD model has already extracted enough information from the image to perform the additional classification task. We test this hypothesis by using a standard classification head and investigate which feature levels provide the best suited information for this task. The classification head consists of two fully connected layers followed by a 19-way Softmax layer.

The four different topologies shown in Figure 5 were implemented. To preserve the previous SOD performance, only the newly added portions shown in blue are changed during the training phase. In Node 1 of Figure 5a, the top layer of the image feature extraction layer is used similar to traditional CNN classifiers. Meanwhile, in Node 2 and 4, we mimic the YOLOv3 multi scale detection method by concatenating the three image feature levels. In Node 4 of Figure $5 \mathrm{~d}$ we include additional residual blocks that can extract further features directly driven by the classification loss. This is extremely costly when considering the amount of additional parameters required. In Node 3 of Figure $5 \mathrm{c}$ we use the object detection features rather than image features since the local features of explicit objects might be strongly linked to the nature of the pornographic content.

In the first step of the training process, the original YOLOv3 architecture with SOD weights was initiated. In order to make sure that the old object detection performance is preserved, all of the model layers were 'frozen' such that the parameter values do not change during any training phase. After adding the new classification node, we assign it's output layer with a categorical loss function whilst giving the three original object detection outputs of '(b)' in Figure 4 'None' values. The 'None' values indicate that no loss will be calculated for that output during the training phase. A validation split of 0.15 was used along with a batch size of 64 along with an 'Adam' optimiser. The training duration depended on an early stopping mechanism which was set to finish the training process if the validation loss does not improve after 16 epochs. The learning rate was also reduced by a factor of 0.1 if the validation loss does not improve after 5 epochs. 


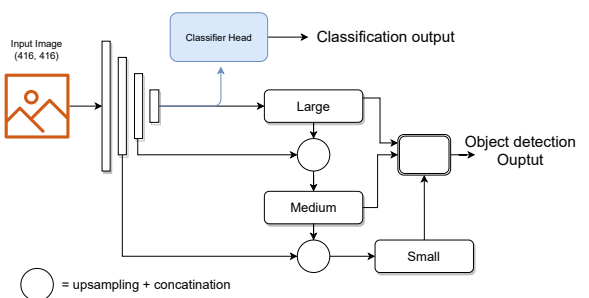

(a) Node 1

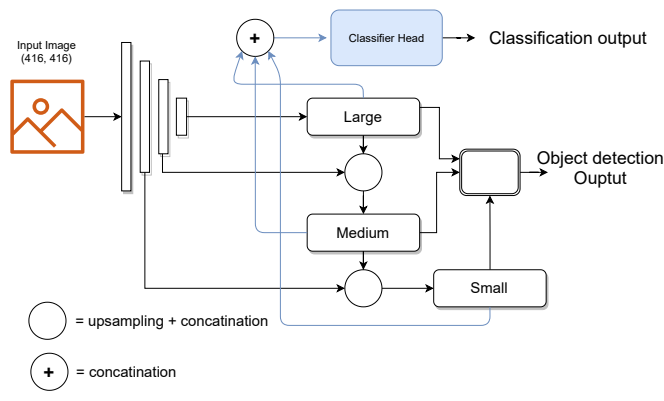

(c) Node 3

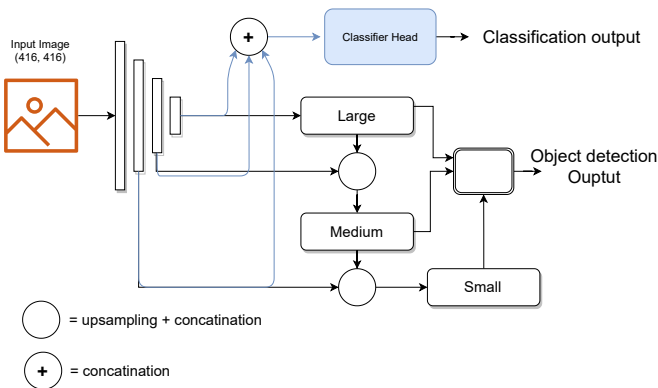

(b) Node 2

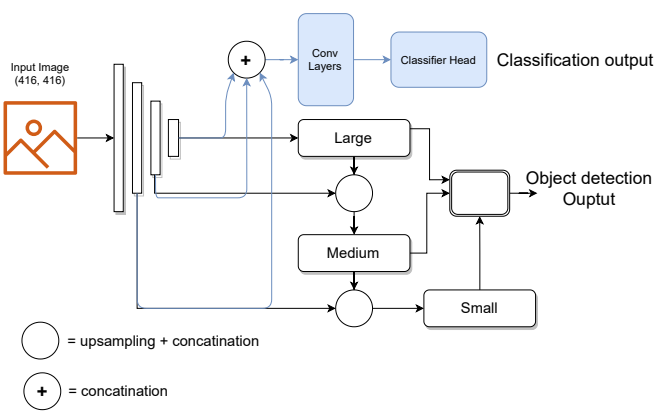

(d) Node 4

Figure 5: Classifier node topologies

\section{RESULTS}

In this section we evaluate our sexual organ detector under three different scenarios. We first show the improvements obtained through data augmentation and multiple confidence thresholding by testing on our custom test set. Here, both the model's object localisation and classification capabilities are addressed using confusion matrices and mean average precision (mAP) score. Following this, we check the applicability of our model to adult pornography image classification using the NPDI-800 test set [3]. In Section 4.3, we analyse this model's performance when processing actual CSA material with the help of the Malta Police Force (MPF). Finally, we evaluate the classification performance of the network topologies described in Section 3.5 using the top-3 accuracy obtained on the test set described in Section 3.2. A MobileNetV2 architecture which was trained end-to-end with the same data will be used as a reference to compare our results with those of a dedicated standalone classifier.

\subsection{Object-detector Performance evaluation}

The Intersection over Union (IoU) method is used to compare the predicted and ground truth objects where we set a minimum overlap of $50 \%$ as a requirement for a prediction to be considered as correct. Using this IoU, mAP scores (mAP-50) are used to quantify the performance of each of the trained models on our custom test set. This test set is made up of 1050 pornographic images containing 2662 labels of sexual organs. Table 4 shows how the data augmentation, fine-tuning and multi-confidence thresholding methods described in Section 3.4 affect the model's performance. A manual parametric
Table 4: Sexual organ detection model performance when evaluated on our custom test set of 1050 images

\begin{tabular}{llccccc}
\hline Data Aug & $\begin{array}{l}\text { Frozen } \\
\text { Layers }\end{array}$ & $\begin{array}{c}\text { Confidence } \\
\text { Threshold }\end{array}$ & Alpha & mAP & FPs & FNs \\
\hline No & 0 & 0.2 & & 57.36 & 242 & 287 \\
Yes & 185 & 0.2 & & 47.00 & 329 & 507 \\
Yes & 0 & 0.25 & & 59.82 & 158 & 380 \\
Yes & 0 & Multi & 0.20 & 53.85 & $\mathbf{1 2 5}$ & 463 \\
Yes & 0 & Multi & 0.50 & 61.49 & 229 & 305 \\
Yes & 0 & Multi & 0.80 & $\mathbf{6 3 . 6 3}$ & 333 & $\mathbf{2 2 9}$ \\
\hline
\end{tabular}

FNs: False Positives

Aug: Augmentation

Multi: Set of confidences per class generated using corresponding Alpha value indicated in the same row

search was performed to find the optimal confidence threshold for each model at intervals of 0.05 before tabulating the results.

Table 4 indicates that the best model is obtained through endto-end training on our augmented data with multiple confidence thresholds, generated at a high $\alpha$-value of 0.8 . It becomes evident that when using a low $\alpha$-value, the precision of the model is favoured through higher confidence scores for each class. This decreases the number of false positives as is also shown in Table 4. In general, we note that the YOLOv3 object detection model displays a high resilience against false positives, even at lower confidence ranges as evident by the $158(<6 \%)$ false positive values at a confidence threshold of 0.25 . Increasing the $\alpha$-value favours a lower recall value, hence increasing the overall $\mathrm{mAP}$ score $3.81 \%$ from $59.82 \%$ to $63.63 \%$. This is comparable to the mAP score of $63.5 \%$ achieved by Mallmann's Private Part Detector (PPCensor) [19]; 


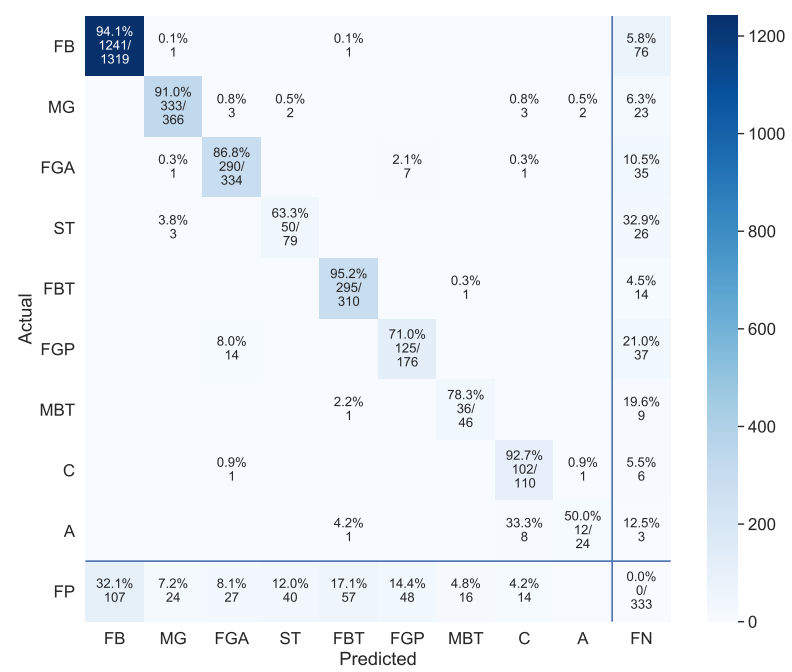

(a)

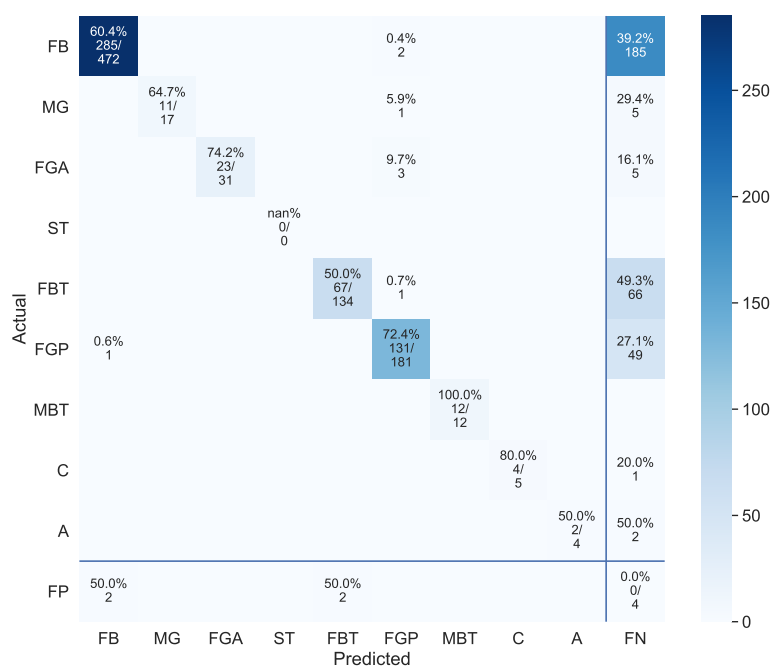

(b)

Figure 6: Confusion matrices obtained from; (a) 1050 Adult pornography images with, (b) 609 Child sexual abuse images. The class names are abbreviated as indicated in the first column of Table 2.
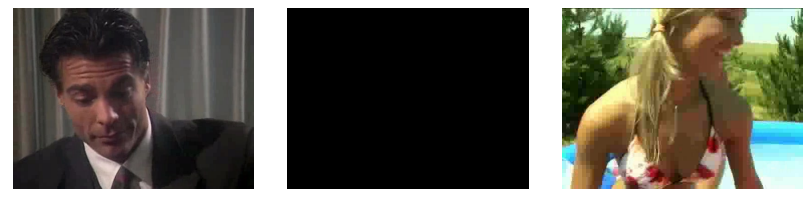

Figure 7: Examples of non pornographic frames extracted from the pornographic class of NPDI-800

however, the latter only discriminated between 4 classes compared to the 9 classes distinguished here. Besides detecting more than twice as many classes, our framework also displays an average throughput of 29.2 frames per second (FPS) on a Nvidia RTX2080-Ti GPU, roughly 2.7 times faster than the PPCensor which reportedly performed at $10.86 \mathrm{FPS}$.

Figure 6 a shows the confusion matrix displaying a strong diagonal obtained by the model operating at with multiple confidence thresholds based on an $\alpha$-value of 0.8 . The only considerable classification confusion took place between the "Coitus" and "Anal" classes where $33 \%$ of "Anal" annotations where predicted as "Coitus". This confusion is understandable since these two action classes present similar human poses, further aggravated by partial occlusions.

\subsection{Adult pornography Classification}

Our best performing model was also used as an image classifier whereby an image is considered as pornographic if it contains at least one sexual organ. The NPDI-800 pornography dataset has been used to evaluate multiple models to detect pornography in videos [2], most of which perform frame level classification. Consisting of 400 pornographic, 200 benign and 200 non-porn ("difficult") videos the NPDI-800 dataset amounts to 77 hours of content. The middle frame of each video shot is used to obtain a total of 16,727 frames, of which: 6,785 are benign; 3,555 are non-pornographic, but difficult to distinguish; and 6,387 are obtained from pornographic videos. As shown by the examples shown in Figure 7, not all the frames in pornographic videos are necessarily explicit. Thus, we manually filtered the pornographic set and made sure that each image contains adult pornography content. As a result, we selected 3,720 pornographic images alongside the 10,350 original benign images. On these images, a frame level accuracy of $87.7 \%$ was obtained but a high false negative rate of $42.04 \%$ was observed. The NPDI800 image sizes are considerably lower than those found in our training data, which may have caused sexual organs to be missed. We use our custom test set of 1050 pornographic images utilised in Section 4.1 to compare this false positive rate to that obtained on stand alone pornographic images. A lower false negative rate of $2.47 \%$ was obtained showing that the low quality of the NPDI video frame images effects our model's performance. Since we operate mainly on local contexts, blurriness of such areas may cause sexual organs to be missed. Meanwhile, the low false positive rate of $2.98 \%$ observed on the video frames shows that model is resilient against mistaking benign instances with sexual content.

Since the NPDI dataset is designed to serve as a video classification dataset, we adopt Tran et al.'s [30] video classification method which is similarly based on explicit object detection. A frame for every second of each video in the dataset is extracted to serve as a key-frame. If at least one sexual organ was detected within a frame, that frame was considered pornographic. Meanwhile, a video is considered pornographic if the number of explicit frames exceeds the porn-frame-threshold. This threshold value is calculated through 


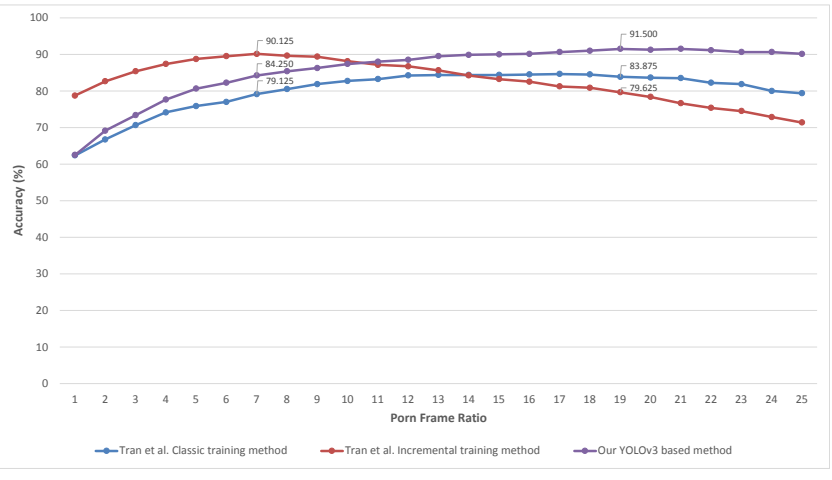

Figure 8: NPDI-800 Video Classification results compared to Tran et al.'s work [30]

Equation 2 for each individual video and as done by Tran et al. [30], we perform a parameter sweep for the optimal pornographic frame ratio (PFR) variable described in Equation 2.

$$
\text { Porn Frame Ratio }=\frac{\text { porn frame threshold }}{\text { total key frames in video }}
$$

Figure 8 shows that our model reaches a video classification accuracy of $91.5 \%$ at an optimal PFR of $19 \%$, outperforming Tran et al.'s work by $1.24 \%$ when comparing directly with their optimal performance at a PFR of $7 \%$.

\subsection{CSA Performance}

Conforming to the long term objective of detecting CSA material, we test our model on images containing CSA material with the help of the MPF's Cyber-crime unit. For this purpose, we packaged our detector into a stand-alone application such that we - as research scientists - never possessed any CSA material. An $\alpha$-value of 0.5 to get an unbiased idea of the model's precision and recall on CSA material. This application was used by a police inspector trained in the detection of CSA material, to collect performance data on an unlabelled set of $384 \mathrm{CSA}$ images. In this test our model displayed a sexual organ detection precision of $97.81 \%$ and a recall of $63.09 \%$. The confusion matrix obtained is shown in Figure $6 \mathrm{~b}$ alongside the confusion matrix generated from our 1050 adult pornography image test set. When compared to the adult pornography confusion matrix, a strong diagonal can still be observed but it becomes evident that the number of false negatives increased considerably. This suggests that the model's performance can be improved by prioritising the recall through increasing the $\alpha$-value.

For CSA image classification, our model achieves a true positive rate of $75.3 \%$. An image was flagged when at least one sexual organ was detected, resulting in 295 of the 384 CSA images containing sexual organs being flagged.

\subsection{Pornography Description Classification}

Table 5 displays the top-3 accuracy achieved by each model variation. The MobileNet set the baseline benchmark at $88 \%$ top-3 accuracy when using 2.6 million additional parameters. Thus, even though the best accuracy was achieved with the addition of node 4 ,
Table 5: Classification results from each model topology

\begin{tabular}{lrr}
\hline Model & Number of Trainable Parameters & Top-3 Accuracy \\
\hline MobileNetV2 & $2,637,799$ & 0.88 \\
SOD + Node 1 & $\mathbf{2 9 7 , 7 4 7}$ & 0.878 \\
SOD + Node 2 & 494,355 & 0.83 \\
SOD + Node 3 & 627,987 & 0.868 \\
SOD + Node 4 & $11,282,195$ & $\mathbf{0 . 9 0 4}$ \\
\hline
\end{tabular}

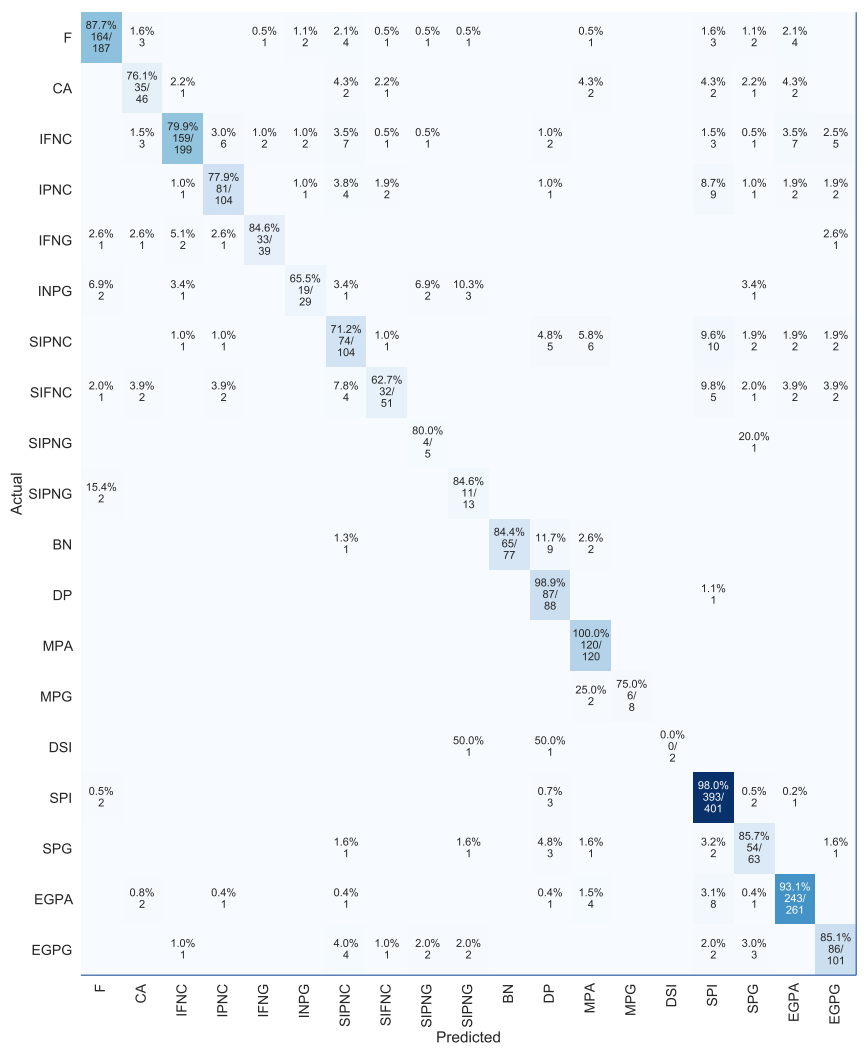

Figure 9: Classifier Confusion Matrix

including additional residual blocks was shown to be too computationally expensive. Meanwhile, using just the final image feature layer (node 1), we manage to match the MobileNet's classification performance with just a tenth of the additional parameters.

The overlap between a large number of pornographic scenarios is inevitable where classes that contain group activities might contain multiple instances of 'couple' or 'alone' acts such as 'Fellatio' and 'Exposed Genitals Posing Alone'. Thus, we still consider the top3 class predictions even when generating the confusion matrix shown in Figure 9. We note that most misclassifications are caused by confusion between closely related classes or the model detects another class scenario which was not set as the ground truth label.

\section{CONCLUSION}

To aid Law Enforcement Agency (LEA) detect and rank both adult and child pornography, we propose using a sexual organ detector. 
This approach aims to reduce false positive flagging rates by directly detecting the content that makes images explicit. In this work, we present a framework that achieves a mean average precision (mAP) score of $63.63 \%$ on adult pornographic material, and a sexual organ detection precision of $97.81 \%$ and recall of $63.09 \%$ on CSA material. The very high precision shows that this framework is capable of generating a negligible volume false positives. It was shown that the features learnt by the sexual organ detector are also applicable to CSA material in Section 4, where a strong confusion matrix diagonals were obtained even though the training data consisted of just adult pornographic material. These feature extractors were also shown to be informative enough to distinguish between different pornographic scenarios with a top-3 accuracy of $87.8 \%$. The concept used here can be applied to different domains, where a strong image feature extraction base is used with different heads that reuse information to perform multiple tasks in a single model, increasing the efficiency of a system as a whole. We aim to decrease the number of false negatives by working with the MPF to fine tune our model on a number of labelled images containing CSA material.

\section{ACKNOWLEDGEMENTS}

This research has been funded with support from the European Commission under the 4NSEEK project with Grant Agreement 821966. This publication reflects the views only of the authors, and the European Commission cannot be held responsible for any use which may be made of the information contained therein.

\section{REFERENCES}

[1] Waleed Abdulla. 2017. Mask R-CNN for object detection and instance segmentation on Keras and TensorFlow. https://github.com/matterport/Mask_RCNN.

[2] Nouar AlDahoul, Hezerul Abdul Karim, Mohd Haris Lye Abdullah, M.F.A. Fauzi, A.S.B. Wazir, S. Mansor, and J. See. 2020. Transfer Detection of YOLO to Focus CNN's Attention on Nude Regions for Adult Content Detection. Symmetry 13, 1 (dec 2020), 26. https://doi.org/10.3390/sym13010026

[3] Sandra Avila, Nicolas Thome, Matthieu Cord, E. Valle, and A.A. Araújo. 2013 Pooling in image representation: The visual codeword point of view. Computer Vision and Image Understanding 117, 5 (may 2013), 453-465. https://doi.org/10. 1016/j.cviu.2012.09.007

[4] Raffaella Bernardi, Ruket Cakici, Desmond Elliott, A. Erdem, E. Erdem, N. IkizlerCinbis, F. Keller, A. Muscat, and B. Plank. 2016. Automatic Description Generation from Images: A Survey of Models, Datasets, and Evaluation Measures. Journal of Artificial Intelligence Research 55 (feb 2016), 409-442. https://doi.org/10.1613/jair. 4900

[5] Roderic Broadhurst. 2019. Child sex abuse images and exploitation materials. In The Human Factor of Cybercrime. Routledge, 310-336. https://doi.org/10.4324/ 9780429460593-14

[6] Jacob Devlin, Hao Cheng, Hao Fang, Saurabh Gupta, Li Deng, Xiaodong He, Geoffrey Zweig, and Margaret Mitchell. 2015. Language Models for Image Captioning: The Quirks and What Works. (2015) arXiv:http://arxiv.org/abs/1505.01809v3 [cs.CL]

[7] Mohd Saad Hamid, NurulFajar Abd Manap, R.A. Hamzah, and A.F. Kadmin 2020. Stereo matching algorithm based on deep learning: A survey. fournal of King Saud University - Computer and Information Sciences (aug 2020). https: //doi.org/10.1016/j.jksuci.2020.08.011

[8] M. Hodosh, P. Young, and J. Hockenmaier. 2013. Framing Image Description as a Ranking Task: Data, Models and Evaluation Metrics. Fournal of Artificial Intelligence Research 47 (aug 2013), 853-899. https://doi.org/10.1613/jair.3994

[9] Mohammad Hosein Hamian, Ali Beikmohammadi, Ali Ahmadi, and Babak Nasersharif. 2021. Semantic Segmentation of Autonomous Driving Images by the Combination of Deep Learning and Classical Segmentation. In 2021 26th International Computer Conference, Computer Society of Iran (CSICC). 16. https://doi.org/10.1109/CSICC52343.2021.9420573

[10] Lizhi Huang and Xunyi Ren. 2018. Erotic image recognition method of bagging integrated convolutional neural network. In Proceedings of the 2nd International Conference on Computer Science and Application Engineering (CSAE '18). ACM, Article 107, 7 pages. https://doi.org/10.1145/3207677.3277990
[11] X. Kejun, W. Jian, N. Pengyu, and H. Jie. 2012. Automatic nipple detection using cascaded AdaBoost classifier. In 2012 Fifth International Symposium on Computational Intelligence and Design, Vol. 2. 427-432. https://doi.org/10.1109/ ISCID.2012.262

[12] Ryan Kiros, Ruslan Salakhutdinov, and Richard S. Zemel. 2014. Unifying Visual-Semantic Embeddings with Multimodal Neural Language Models. (2014). arXiv:http://arxiv.org/abs/1411.2539v1 [cs.LG]

[13] Girish Kulkarni, Visruth Premraj, Vicente Ordonez, Sagnik Dhar, Siming Li, Y. Choi, A.C. Berg, and T.L. Berg. 2013. BabyTalk: Understanding and Generating Simple Image Descriptions. IEEE Transactions on Pattern Analysis and Machine Intelligence 35, 12 (dec 2013), 2891-2903. https://doi.org/10.1109/tpami.2012.162

[14] Rémi Lebret, Pedro O. Pinheiro, and Ronan Collobert. 2015. Phrase-based Image Captioning. (2015). arXiv:http://arxiv.org/abs/1502.03671v2 [cs.CL]

[15] Hee-Eun Lee, Tatiana Ermakova, V Ververis, and B Fabian. 2020. Detecting child sexual abuse material: A comprehensive survey. Forensic Science International: Digital Investigation 34 (sep 2020), 301022. https://doi.org/10.1016/j.fsidi.2020. 301022

[16] Rainer Lienhart and Jochen Maydt. 2002. An extended set of haar-like features for rapid object detection. In Proceedings. International Conference on Image Processing, Vol. 1. IEEE, I-I.

[17] Bee Lim, Sanghyun Son, Heewon Kim, Seungjun Nah, and Kyoung Mu Lee. [n.d.]. Enhanced Deep Residual Networks for Single Image Super-Resolution. ([n. d.]). arXiv:1707.02921v1 [cs.CV]

[18] J. Mahadeokar and G. Pesavento. 2016. Open sourcing a deep learning solution for detecting nsfw images. Available online: https://yahooeng.tumblr.com/post/151148689421/open-sourcing-a-deeplearning-solution-for.

[19] Jackson Mallmann, Altair Olivo Santin, E.K. Viegas, R.R Santos, and J Geremias. 2020. PPCensor: Architecture for real-time pornography detection in video streaming. Future Generation Computer Systems 112 (nov 2020), 945-955. https: //doi.org/10.1016/j.future.2020.06.017

[20] Ethel Quayle Maxwell Taylor. 2003. Child Pornography: An Internet Crime. https: //bit.ly/2TkpfSQ

[21] Microsoft. [n.d.]. Visual Object Tagging Tool (VoTT). https://github.com/ microsoft/VoTT

[22] M. N. Moustafa. 2015. Applying deep learning to classify pornographic images and videos. In 7th Pacific-Rim Symposium on Image and Video Technology (PSIVT).

[23] University of Leon, Group for Vision, and Intelligent systems (GVIS). 2019. Adult Pornography Dataset 2M. Online. http://gvis.unileon.es/dataset/apd-2m/

[24] Omkar M. Parkhi, Andrea Vedaldi, and Andrew Zisserman. 2015. Deep Face Recognition. In Procedings of the British Machine Vision Conference 2015. British Machine Vision Association. https://doi.org/10.5244/c.29.41

[25] Joseph Redmon and Ali Farhadi. 2018. YOLOv3: An Incremental Improvement. arXiv:1804.02767 [cs.CV]

[26] Shaoqing Ren, Kaiming He, Ross Girshick, and Jian Sun. 2016. Faster RCNN: Towards Real-Time Object Detection with Region Proposal Networks. arXiv:1506.01497 [cs.CV]

[27] Mark Sandler, Andrew Howard, Menglong Zhu, A. Zhmoginov, and L.C. Chen. [n.d.]. MobileNetV2: Inverted Residuals and Linear Bottlenecks. ([n.d.]). arXiv: $1801.04381 \mathrm{v} 4$ [cs.CV]

[28] Rongbo Shen, Fuhao Zou, Jingkuan Song, Kezhou Yan, and Ke Zhou. 2018. EFUI: An ensemble framework using uncertain inference for pornographic image recognition. Neurocomputing 322 (dec 2018), 166-176. https://doi.org/10.1016/j.neucom. 2018.08.080

[29] Andre Tabone, Alexandra Bonnici, Stefania Cristina, Reuben Farrugia, and Kenneth Camilleri. 2020. Private Body Part Detection using Deep Learning. Volume1:ICPRAM, 20511 (2020).

[30] Hoang-Loc Tran, Quang-Huy Nguyen, Dinh-Duy Phan, Thanh-Thien Nguyen, K.N.K. Nguyen, and D.L. Vu. 2020. Additional Learning on Object Detection: A Novel Approach in Pornography Classification. In Future Data and Security Engineering. Big Data, Security and Privacy, Smart City and Industry 4.0 Applications. 311-324. https://doi.org/10.1007/978-981-33-4370-2_22

[31] Paulo Vitorino, Sandra Avila, Mauricio Perez, and Anderson Rocha. 2018. Leveraging deep neural networks to fight child pornography in the age of social media. Journal of Visual Communication and Image Representation 50 (2018), 303 - 313. https://doi.org/10.1016/j.jvcir.2017.12.005

[32] Yuhui Wang, Xin Jin, and Xiaoyang Tan. 2016. Pornographic image recognition by strongly-supervised deep multiple instance learning. In 2016 IEEE International Conference on Image Processing (ICIP). IEEE. https://doi.org/10.1109/icip.2016. 7533195

[33] Yue Wang, Jun Li, Hee Lin Wang, and Zujun Hou. 2010. Automatic nipple detection using shape and statistical skin color information. In $M M M$.

[34] J. Wehrmann, G. S. Simões, R. C. Barros, and V. F. Cavalcante. 2018. Adult content detection in videos with convolutional and recurrent neural networks. Neurocomputing 272 (2018), 432-438.

[35] Z. Yong-Xia and Z. Ge. 2010. MD5 Research. In 2010 Second International Conference on Multimedia and Information Technology, Vol. 2. 271-273. https: //doi.org/10.1109/MMIT.2010.186 